\title{
Tuberculosis Cutánea
}

\author{
Cutaneous Tuberculosis
}

Gabriela María Rivera Vega ${ }^{1}$ (1) https://orcid.org/0000-0002-7251-4137, Nataly Yamaly Martínez Yanes² ${ }^{2}$ https://orcid.org/0000-0001-9644-1473.

${ }^{1}$ Hospital del Valle; Instituto Hondureño de Seguridad Social, Hospital Regional del Norte; San Pedro Sula, Honduras. ${ }^{2}$ Hospital Tela; Tela, Honduras.

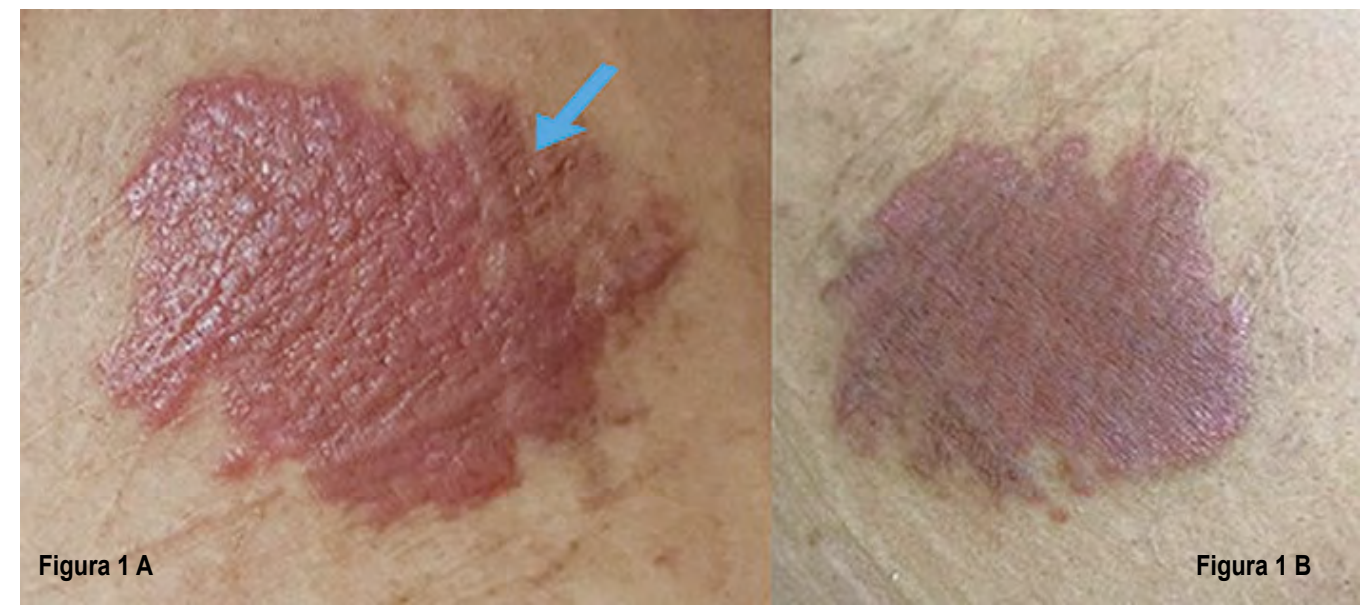

Mujer de 30 años, odontóloga. Presentó mácula, pardo oscuro, de bordes mal definidos y elevados, consistencia firme, 6 × $6.5 \mathrm{~cm}$ de diámetro, indolora, pruriginosa, localizada en glúteo, de 3 meses de evolución. Biopsia reporta: Dermatitis crónica granulomatosa con signos que sugieren tuberculosis cutánea. PPD negativa. Hemograma, Química sanguínea, radiografía de tórax normal. Tuberculosis cutánea: enfermedad infecciosa, causada por Mycobacterium tuberculosis, en piel ocasiona formas fijas y hematógenas, dependiente de la fuente de infección y estado inmunológico del huésped. Existen varios subtipos, Tuberculosis luposa o lupus vulgar: placas eritematoescamosas o verrugosas de crecimiento centrifugo, que pueden ulcerarse en su centro y dejan cicatrices atróficas o queloidales. ${ }^{1}$ (Figura 1A) Diagnóstico histológico: tubérculo compuesto de linfocitos, células epiteloides y células gigantes multinucleadas tipo Langhans, correspondiente al granuloma tuberculoide. Existen grados de necrosis caseosa y lesiones vasculares. Diagnóstico diferencial: leishmaniasis, sarcoidosis, esporotricosis fija, lupus eritematoso discoide, sífilis terciaria, lepra, carcinoma de células escamosas, liquen plano hipertrófico y otros subtipos de tuberculosis cutánea. El tratamiento que el Centro de Control de Enfermedades (CDC) propone: isoniacida, rifampicina, pirazinamida y etambutol, diariamente por 2 meses, luego isoniacida y rifampicina bisemanal por 4 meses. ${ }^{2}$ La lesión disminuyó de tamaño, posterior a 4 meses del tratamiento (Figura 1B).

Recibido: 26-03-2021 Aceptado: 05-09-2021 Primera vez publicado en línea: 05-10-2021 Dirigir correspondencia a: Gabriela María Rivera Vega

Correo electrónico: gamarive2306@gmail.com

\section{RELACIONES Y ACTIVIDADES FINANCIERAS Y NO FINANCIERAS: Ninguno}

\section{DECLARACIÓN DE CONFLICTOS DE INTERÉS: Ninguno}

Forma de citar: Rivera Vega GM, Martínez Yanes NY. Tuberculosis cutánea. Rev Méd Hondur. 2021; 89(2): 141. DOI: https://doi.org/10.5377/rmh.v89i2.12528

(C) 2021 Autor(es). Artículo de acceso abierto bajo la licencia https://creativecommons.org/ licenses/by/4.0/deed.es

\section{DETALLES DE AUTOR(ES)}

Gabriela María Rivera Vega, Médica General; gamarive2306@gmail.com Nataly Yamaly Martínez Yanes, Médica General; martineznataly171@ gmail.com

\section{REFERENCIAS}

1. Tincopa Wong O, Sánchez-Saldaña L. Tuberculosis cutánea. Dermat Perú [Internet]. 2003. [citado 26 febrero 2021];13(3):195-214 Disponible en: https://sisbib.unmsm.edu.pe/Bvrevistas/dermatologia/v13_n3/Pdf/a06.pdf

2. Centros para el Control y la Prevención de Enfermedades. Tratamiento para la enfermedad de la tuberculosis [Internet]. Atlanta: CDC; 2016. [citado 15 enero 2021]. Disponible en: https://www.cdc.gov/tb/esp/topic/ treatment/tbdisease.htm 